Case Report

\title{
Horizontal Ridge Augmentation Using a Xenograft Bone Substitute for Implant-Supported Fixed Rehabilitation: A Case Report with Four Years of Follow-Up
}

\author{
Bruno Freitas Mello, ${ }^{1}$ Márcio de Carvalho Formiga ${ }^{(D)},{ }^{1}$ Luiz Fernando de Souza da Silva, ${ }^{2}$ \\ Gustavo dos Santos Coura, ${ }^{2}$ and Jamil Awad Shibli ${ }^{1}$ \\ ${ }^{1}$ Department of Periodontology and Oral Implantology, Dental Research Division, University of Guarulhos, Sao Paulo, Brazil \\ ${ }^{2}$ Department of Implantology, Unisociesc, Florianópolis, Brazil
}

Correspondence should be addressed to Márcio de Carvalho Formiga; marciocformiga@gmail.com

Received 18 April 2019; Accepted 2 May 2020; Published 15 May 2020

Academic Editor: Mine D ndar

Copyright (c) 2020 Bruno Freitas Mello et al. This is an open access article distributed under the Creative Commons Attribution License, which permits unrestricted use, distribution, and reproduction in any medium, provided the original work is properly cited.

\begin{abstract}
The guided bone regeneration (GBR) technique has been used to achieve optimal bone volume augmentation and allow dental implant placement in atrophic maxilla and mandible, with predictable results and high survival rates. The use of bone substitutes has reduced the necessity of autogenous bone grafts, reducing the morbidity at the donor areas and thus improving the patients' satisfaction and comfort. This clinical case report shows a clinical and histological evaluation of the bone tissue behavior, in a case that required the horizontal augmentation of the alveolar ridge, with the use of xenograft biomaterial and further dental implant placement. After six months of healing time, six implants were placed, and a bone biopsy was done. The histological analysis depicted some fragments of the xenograft bone graft, integrated with the new-formed bone tissue.
\end{abstract}

\section{Introduction}

The rehabilitation of totally, partially, and single-unit edentulous patients with the use of dental implants is an established treatment with high success rates $[1,2]$. However, there are several unfavorable clinical situations for the placement of implants, where there is not sufficient bone height and/or width in the alveolar bone ridge. Augmentation of the alveolar ridge using guided bone regeneration (GBR) became a treatment option to obtain bone support for osseointegrated dental implants [3]. The procedures for bone augmentation of the alveolar ridge through GBR present successful, long-term follow-up results [4-6].

Autogenous bone grafts are often used in GBR procedures and are considered as the gold standard because it is the only biomaterial that combines properties of osteogenesis, osteoinduction, and osteoconduction [7, 8]. However, disadvantages such as morbidity at the donor site, limited availability, tooth sensitivity, and risk of dehiscence of the wounds [1, 7, 9-11] have led to investigations on the development and application of bone substitutes for the regeneration of the alveolar bone ridge [11-13].

Xenografts have shown to have excellent properties for GBR, like its biocompatibility, formation of a scaffold (osteoconduction), slow resorption rates, and the ability to define and maintain the volume for bone gain [12-14]. The graft maturation period could be longer than that of autogenous bone grafts, taking from nine to twelve months $[10,12-14]$, and demand the need of collagen membranes for guided bone regeneration procedures, which must provide cell occlusion and a better biocompatibility with the soft tissue, reducing the risk of complications such as wound dehiscence $[11,14]$.

Therefore, the aim of this case report is to describe a GBR with xenograft and collagen membrane for horizontal augmentation for an implant-supported fixed rehabilitation and the four years of follow-up. 

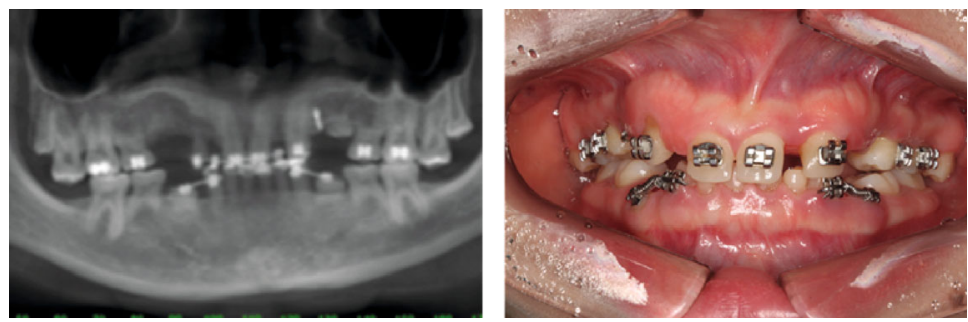

Figure 1: Panoramic radiograph (a) and intraoral buccal view, showing permanent tooth absences and presence of several deciduous teeth.
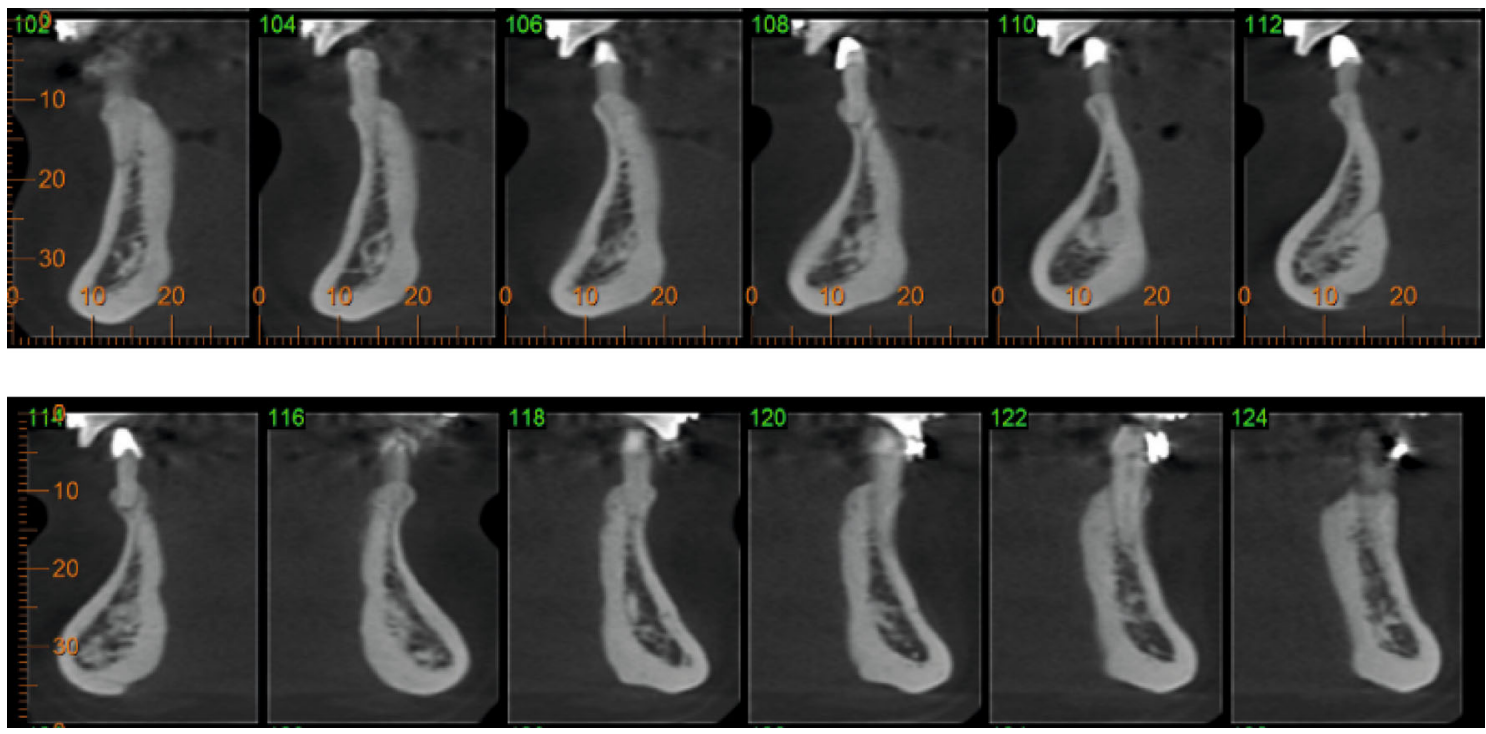

FIGURE 2: Preoperatory tomography of the anterior mandible region.

\section{Case Report}

An 18-year-old female patient attended a clinic/school seeking to solve aesthetic and functional problems. After an initial evaluation, several dental losses and the presence of some deciduous teeth were observed, revealing a multiple agenesis condition. Clinical examination and CT scans were performed. In the mandible, the patient presented all the deciduous teeth, and only permanent teeth present were \#36 and \#46 (Figure 1).

Analyzing the mandible CT scan, it was observed a sizeable horizontal deficiency of the alveolar ridge in the crestal symphysis region. This situation made impossible the placement of implants in the ideal three-dimensional position, requiring previous bone reconstruction (Figure 2).

It was decided to perform horizontal bone augmentation by guided bone regeneration with only a bovine bone substitute (Bio-Oss ${ }^{\circledR}$ Geistlich), because it presents reproducible results with acceptable success rates and low rate of complications, besides providing lower morbidity for the patient. The marginal gingiva was healthy and presented a good width of keratinized tissue. Amoxicillin $500 \mathrm{mg} 1$ every 8 hours for 7 days and $0.12 \%$ chlorhexidine gluconate rinse 3 times a day for 14 days were prescribed, initiating 2 days before surgery. Infiltration anesthesia was performed with $4 \%$ articaine anesthetic in the mental nerve plexus, bilaterally. The incision was intrasulcular in the region from 75 to
85, with small vertical releases at the distal of this incision. A mucoperiosteal flap was raised. At the recipient site, soft tissue debridement and small perforations with 1.3 drills were performed in the cortical bone with the purpose of providing irrigation and blood supply to the bone substitute. At this moment, $1.0 \mathrm{cc}$ of bone substitute (Bio-Oss ${ }^{\circledR}$ Geistlich, large granules) was prepared to be placed at the recipient site and covered with double layer collagen resorbable membrane (Bio-Gide ${ }^{\circledR}$ Geistlich, $25 \mathrm{~mm} \times 25 \mathrm{~mm}$ ). Both bone substitute and collagen membrane were displaced on different recipients, with saline for 10 minutes of hydration prior use. The buccal flap was repositioned, covering all the membrane and avoiding displacement. The suture was performed with simple interrupted and sling sutures using the deciduous teeth as support, with silk 4.0 (Ethicon). After the surgical procedure, a bandage (Micropore ${ }^{\circledR}$ ) was performed to compress the surgical area to diminish possible edema (Figure 3).

Nimesulide $100 \mathrm{mg}$ twice a day for 3 days, paracetamol $750 \mathrm{mg} 3$ times a day (if necessary for pain control), and continuation of the preoperative antibiotic and antiseptic mouthwash protocols were prescribed. The patient was instructed to eat only soft foods, put ice bags on the surgical area for 48 hours, and avoid exercises for 7 days. The sutures were removed after 7 days.

After 12 months, the area of the horizontal bone augmentation was clinically evaluated and through cone-beam CT. After the analysis of the tomography radiograph and initial 


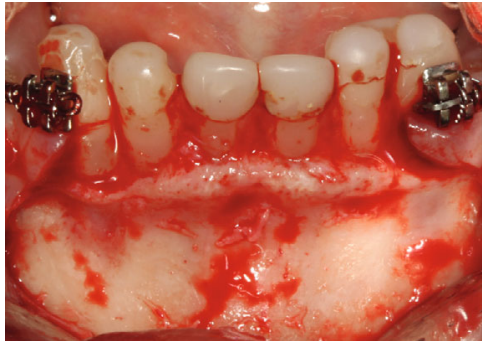

(a)

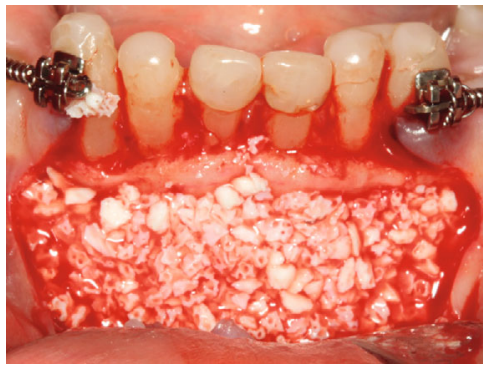

(c)

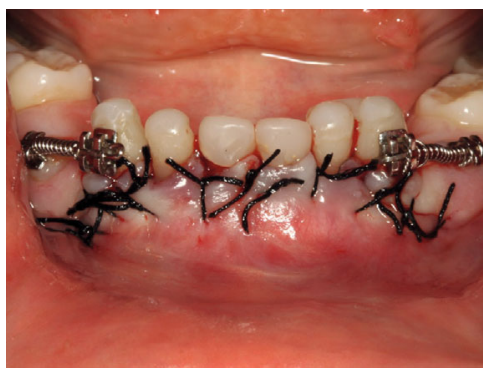

(e)

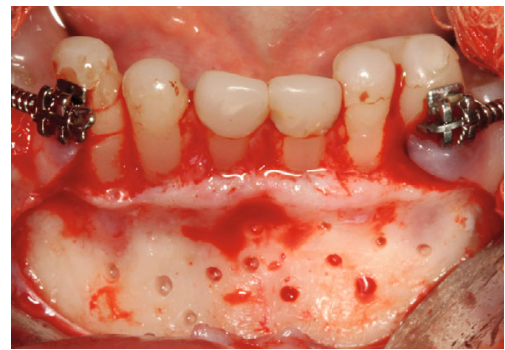

(b)

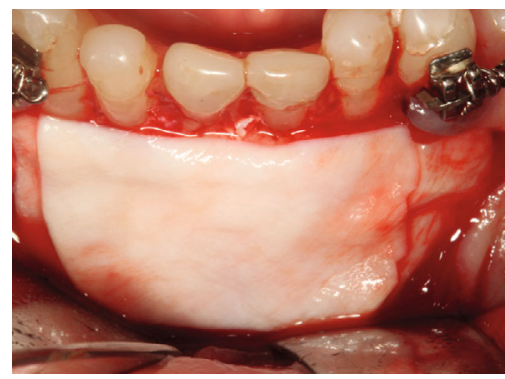

(d)

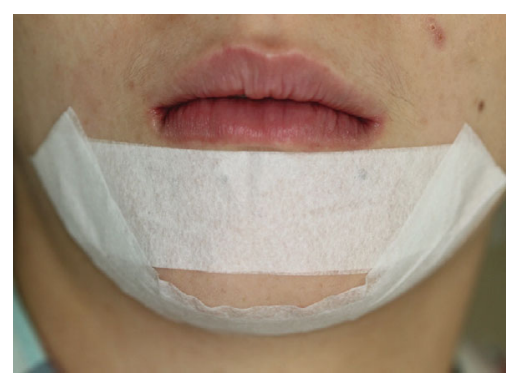

(f)

FIgURE 3: Intraoral view of the surgery steps: (a) incision and total flap detachment, (b) cortical perforations, (c) bone substitute accommodation, (d) double layer collagen membrane, (e) single sutures, and (f) bandage (Micropore ${ }^{\circledR}$ ).

and final prototypes, the gain in bone thickness was approximately $5 \mathrm{~mm}$. Clinically, the success of the procedure was confirmed, as well as the presence of good health of the gingival tissue (Figures 4 and 5).

The surgery to install 6 dental implants $\left(\right.$ Ankylos $^{\circledR}$, Dentsply) was performed 12 months following the horizontal bone augmentation, according to the reverse planning and surgical guide, between the region of \#34 and \#44. All implants presented initial stability above $35 \mathrm{~N} / \mathrm{cm}$. The deciduous teeth $74,73,72,71,81,82,83$, and 84 were removed at this time. After detachment of the mucoperiosteal flap, we found new bone tissue with excellent quality with the native bone appearance and without the presence of loose particles of a bone substitute and surrounded by fibrous tissue. At this moment, a bone fragment of approximately $3 \mathrm{~mm}$ was removed using a $2.0 \mathrm{~mm}$ trephine drill in a buccolingual direction for histological analysis of the grafted area (Figure 6).

In the histological analysis, it was observed the formation of cortical bone with the presence of osteocytes found in several sizes and homogeneously distributed. Bone substitute particles were present in a graft/cortical bone interface. However, it could be confirmed on the clinical observation that the regenerated bone was similar to the natural bone (Figure 7).
Immediately after implant surgery, standard prosthetic abutments (A4) were installed (Ankylos ${ }^{\circledR}$ ). After suturing, with 5.0 polypropylene (Atramat), because of its fewer biofilm accumulation, the impression-taking of the abutments and occlusal registration were accomplished. A temporary acrylic resin fixed prosthesis was installed immediately to the surgical procedure, within 24 hours (Figure 8 ). Pre- and postoperative recommendations and medication protocols were identic to the first surgery, but without the need for bandages.

Four years after bone augmentation of the alveolar ridge, a tomographic examination was requested to evaluate the bone tissue, as well as the implants, where bone normality and stability around the implants were seen, to confirm our clinical evaluation (Figures 9 and 10).

\section{Discussion}

Guided bone regeneration (GBR) performed within the concepts of the technique can be indicated to increase the width of alveolar bone ridges when associated with the use of collagen membranes and bovine bone substitute, with high survival rates of implants placed in sites with large bone volume deficiencies $[6,10,15]$. 


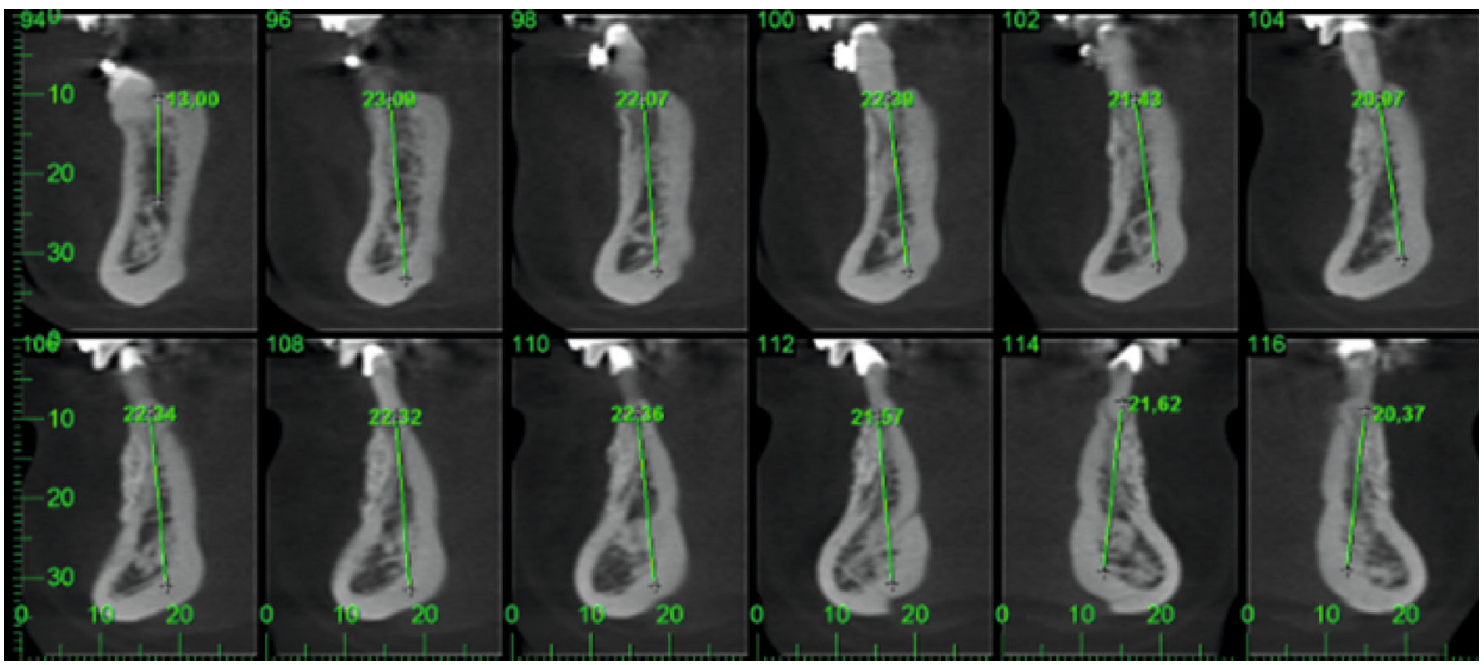

FIgURE 4: Postoperatory tomography radiograph 12 months after the horizontal bone augmentation, showing new bone formation, allowing the correct three-dimensional placement of dental implants.

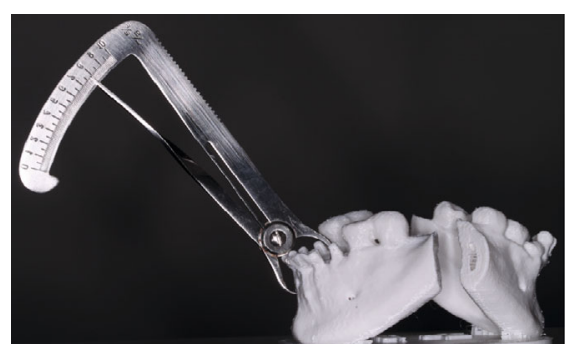

(a)

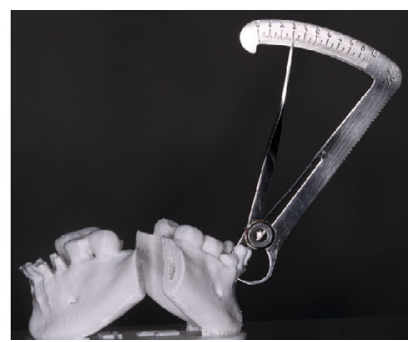

(b)

FIGURE 5: 3D resin prototype of the appearance of the ridge before (a) and after (b) the volume increase of the alveolar ridge. There was a $5 \mathrm{~mm}$ gain in bone thickness.

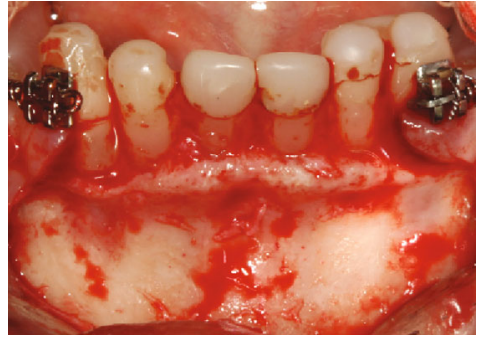

(a)

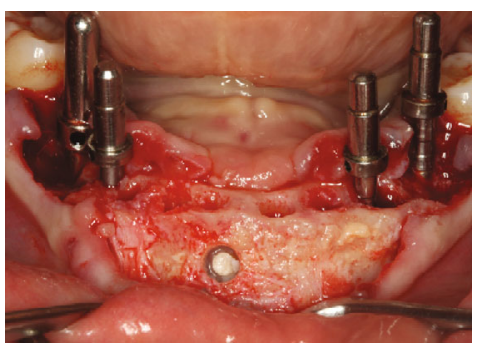

(c)

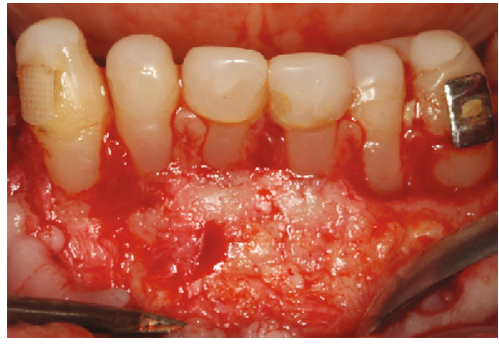

(b)

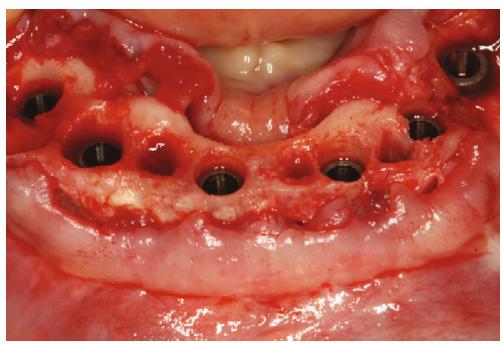

(d)

Figure 6: Intraoral view of the alveolar ridge before (a) and after (b) augmentation. Removal of bone fragment using a $2.0 \mathrm{~mm}$ trephine for histological analysis of the grafted area (c). Six implants in place with initial torque of $35 \mathrm{~N} / \mathrm{cm}(\mathrm{d})$. 


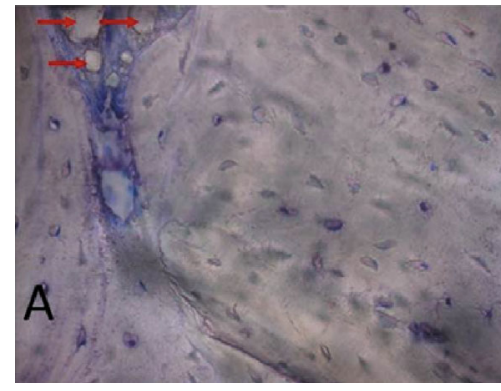

(a)

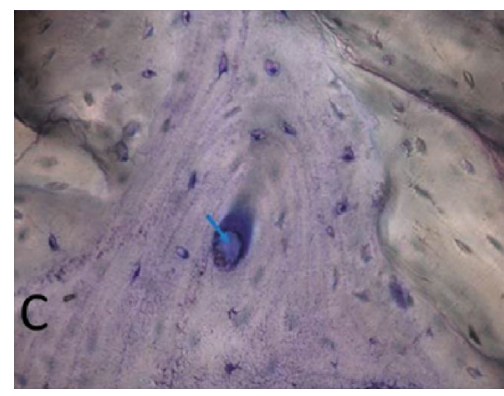

(c)

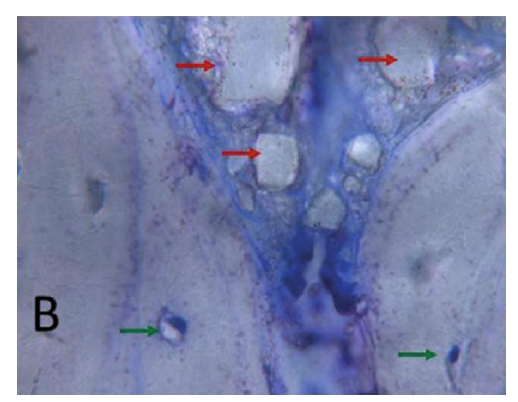

(b)

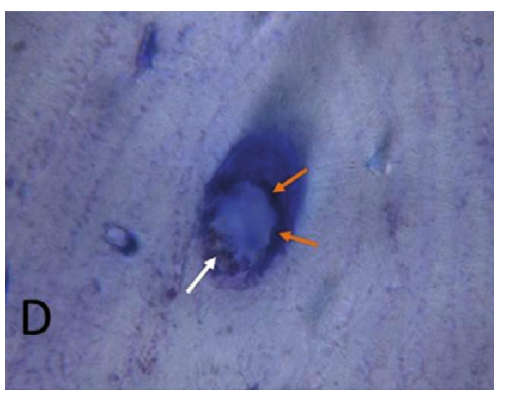

(d)

FIGURE 7: (a) Image of a grafted material interface (red arrow) and cortical bone. It is clearly shown the formation of cortical bone with osteocytes; (b) enlarged image of (a). Grafted material (red arrow). Osteocytes present in the gaps (green arrow); (c) well-formed Haversian system: note the arrangement of concentric lamellae, with the central channel of Havers (blue arrow). Osteocytes homogeneously distributed, demonstrating the usual architecture of cortical bone; (c) enlarged image of (d). In detail, the Havers channel, with endosteum (orange arrow) and red blood cells (white arrow).
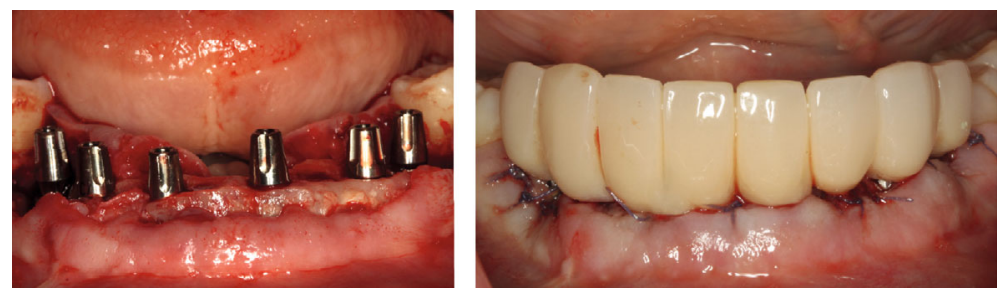

FIGURE 8: Installation of the definitive prosthetic abutments and delivery of the temporary cemented restoration with immediate load.

The horizontal bone gain of about $5 \mathrm{~mm}$ after 12 months observed in this clinical case is within the mean gain obtained by other clinical reports of GBR approach in the literature $[11,12]$. This bone augmentation provided enough bone width for dental implant placement in an ideal threedimensional position.

Soft tissue healing was excellent, with no intercurrences, such as membrane exposure or infections. This corroborated further studies on the compatibility of collagen membranes with soft tissue [9-12]. Studies reported that second intention healing occurred in all membrane exposures, with spontaneous reepithelialization, with no major complications [9].

The collagen absorbable membranes, due to its hydrophilic property and excellent interaction with blood clot fibrin in the surgical wound, facilitate the suture procedure [16] and have less tendency to soft tissue dehiscence [14]. In this case, we used silk suture on the GBR procedure because it was more comfortable for the patient, and the patient had excellent biofilm control. We realize it was not the best suture biomaterial, but the patient was very cooperative during all treatment. On the implant surgery, we preferred the use of polypropylene sutures because it remained above the temporary fixed prosthesis, and this material would provide a better biofilm control. To achieve success in the procedures, GBR membranes must possess functional durability, be resistant to degradation, and have biocompatibility with the soft tissue, providing tissue integration and avoiding spaces that may predispose to tissue dehiscence and infections [15]. The disadvantages of the collagen membrane are the resorption period between 4 and 8 weeks and lack of rigidity, leading to the need for filling material under the membrane thus providing the contour for bone augmentation [16].

Nonabsorbable membranes have greater stability in GBR and are more predictable regarding the time of permanence and maintenance of the scaffold in the alveolar ridge, with excellent results for bone augmentation $[11,16]$. However, some reports have shown that nonresorbable membranes are more critical about their use, leading to high rates of wound dehiscence with subsequent local exposures and infections, and require a second surgery for its often removal $[6,9,14]$. 


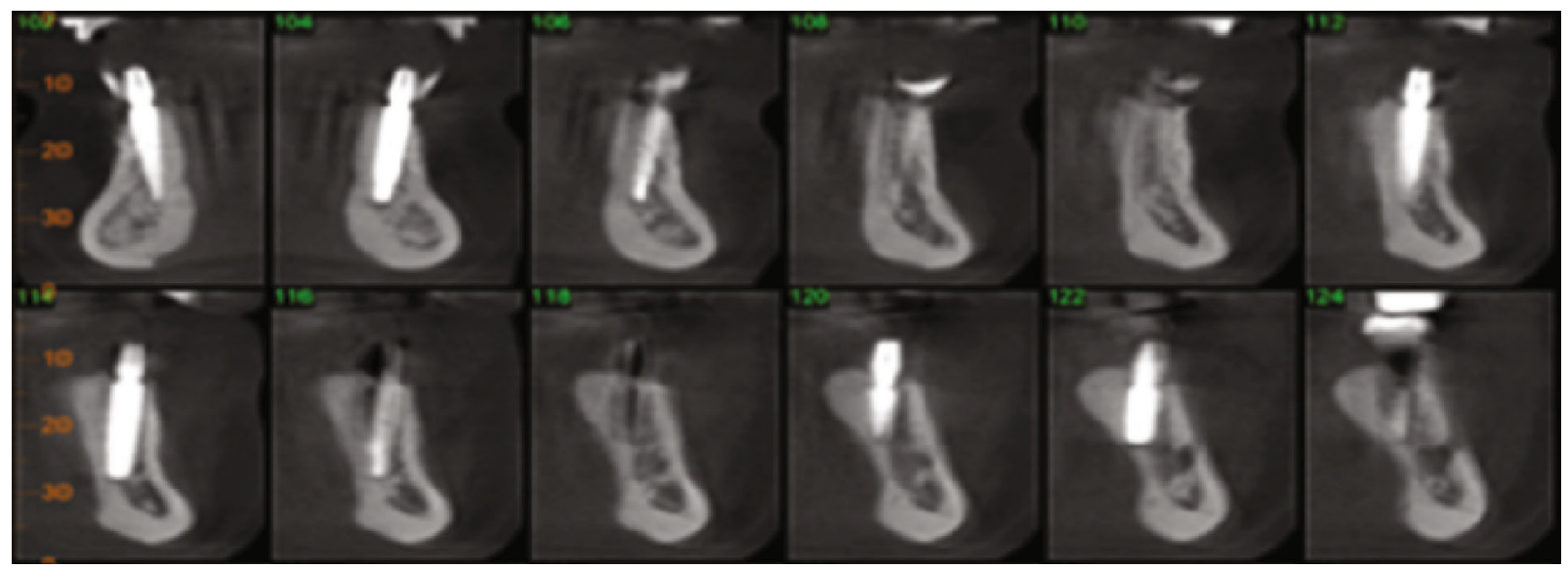

(a)
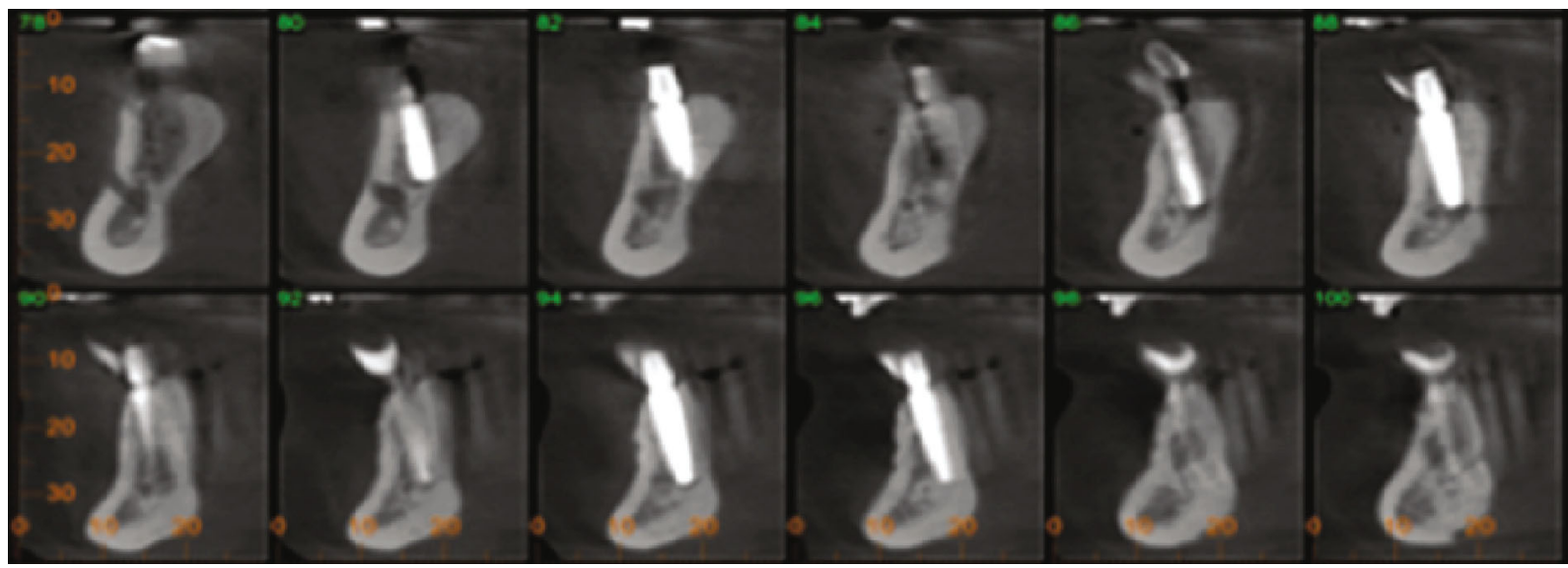

(b)

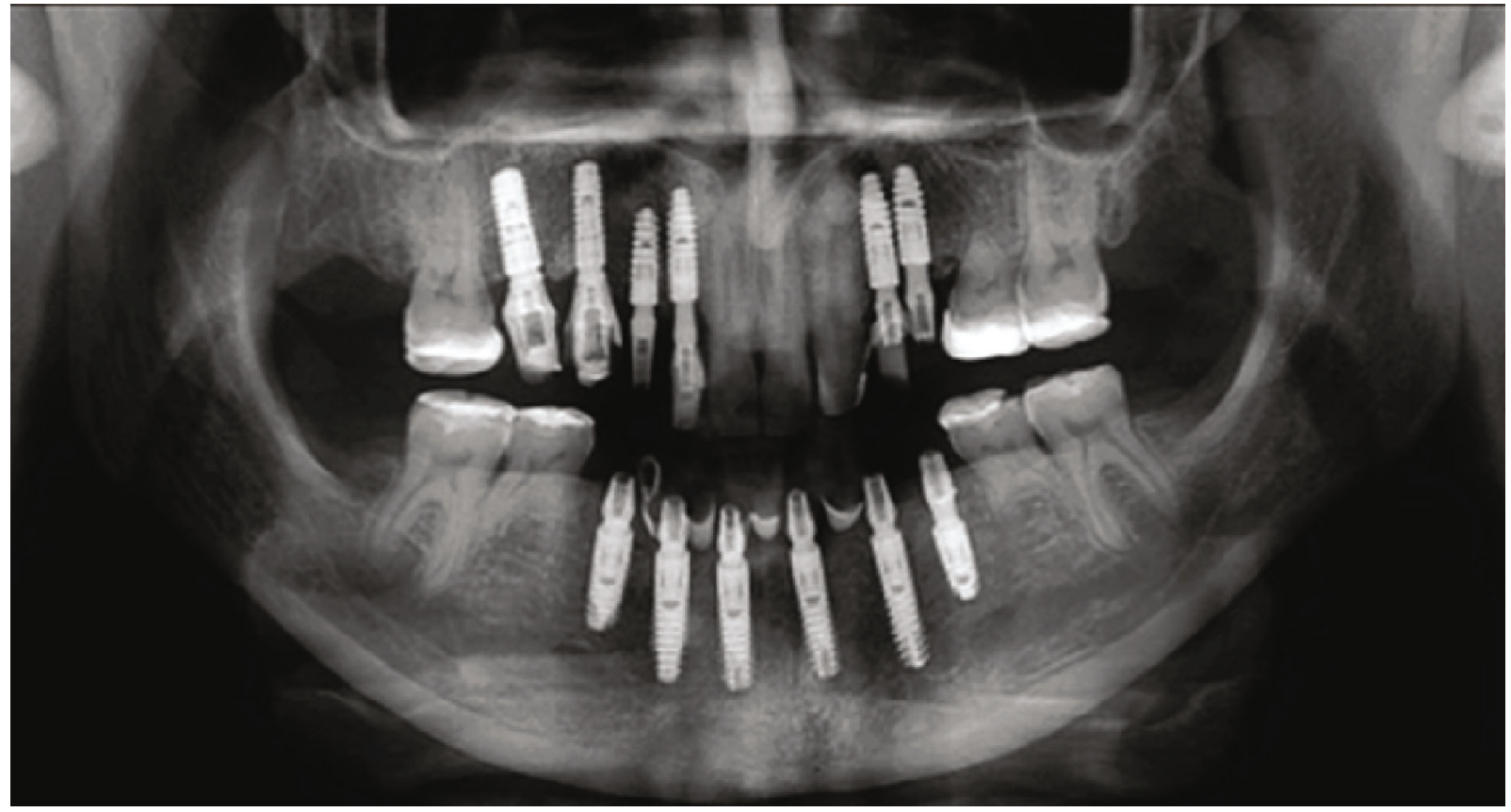

(c)

FIGURE 9: Four-year follow-up CT, showing the stability of the bone tissue around the implants (a, b). Panoramic radiograph of the 4-year follow-up (c). 


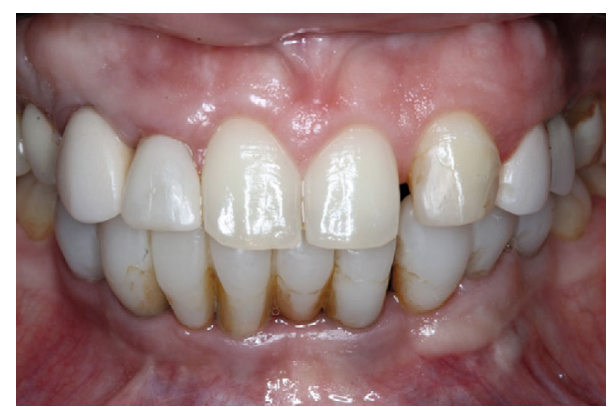

FIgURE 10: Buccal view of the four-year follow-up.

Xenogenous bovine bone substitutes are composed basically of hydroxyapatite, after a process of elimination of all the organic components, resulting in a structure similar to the human bone. Thus, at the end of this process, a biomaterial should be obtained where its particles may have interconnecting pores allowing thereby the penetration of new blood vessels and the osteoblast cellular migration into the graft [13].

In the literature, there are studies that used GBR protocols using collagen membranes and a mixture of autogenous bone/bone substitute [11]. The authors advocate the use of this mixture by the fact that autogenous bone particles add an osteogenic property to the graft.

However, one of the main advantages of the GBR approach using only bone substitutes is to avoid the complications of autogenous bone removal such as donor area morbidity, limited availability, and tooth sensitivity $[1,7,9,10]$. Thus, the use of bone substitutes provides more satisfaction and comfort for the patient. The average results of gains in these studies that used autogenous bone/bone substitute mixture $[10,11]$ are comparable to this clinical case report. Other clinical studies $[17,18]$ showed similar results, as we found in this case report, with high rates of success on implant survival. Recent reviews also endorse the findings of this case report, with similar rates of horizontal bone augmentation [19-21].

\section{Conclusion}

Within the limitations of this report, the horizontal bone augmentation with the use of only a bovine bone substitute and a double layer collagen membrane seemed to be a feasible treatment option in the short term for promoting an increase in bone width of the alveolar ridge, allowing an appropriate size for predictable and successful placement of dental implants and a fixed oral implantsupported rehabilitation.

\section{Data Availability}

The references used to support the findings of this case report are listed in References and can be found at PubMed.

\section{Conflicts of Interest}

The authors declare that there is no conflict of interest regarding the publication of this paper.

\section{References}

[1] B. Al-Nawas and E. Schiegnitz, "Augmentation procedures using bone substitute materials or autogenous bone - a systematic review and meta-analysis," European Journal of Oral Implantology, vol. 7, Supplement 2, pp. S219-S234, 2014.

[2] M. Chiapasco and M. Zaniboni, "Clinical outcomes of GBR procedures to correct peri-implant dehiscences and fenestrations: a systematic review," Clinical Oral Implants Research, vol. 20, Supplement 4, pp. 113-123, 2009.

[3] I. A. Urban, S. A. Jovanovic, and J. L. Lozada, "Vertical ridge augmentation using guided bone regeneration (GBR) in three clinical scenarios prior to implant placement: a retrospective study of 35 patients 12 to 72 months after loading," The International Journal of Oral \& Maxillofacial Implants, vol. 24, no. 3, pp. 502-510, 2009.

[4] T. L. Aghaloo and P. K. Moy, "Which hard tissue augmentation techniques are the most successful in furnishing bony support for implant placement?," The International Journal of Oral \& Maxillofacial Implants, vol. 22, pp. 49-70, 2007.

[5] M. Retzepi and N. Donos, "Guided bone regeneration: biological principle and therapeutic applications," Clinical Oral Implants Research, vol. 21, no. 6, pp. 567-576, 2010.

[6] R. E. Jung, N. Fenner, C. H. F. Hämmerle, and N. U. Zitzmann, "Long-term outcome of implants placed with guided bone regeneration (GBR) using resorbable and non-resorbable membranes after 12-14 years," Clinical Oral Implants Research, vol. 24, no. 10, pp. 1065-1073, 2013.

[7] J. H. Fu and H. L. Wang, "Horizontal bone augmentation: the decision tree," The International Journal of Periodontics \& Restorative Dentistry, vol. 31, no. 4, pp. 429-436, 2011.

[8] M. O. Klein and B. Al-Nawas, "For which clinical indications in dental implantology is the use of bone substitute materials scientifically substantiated?," European Journal of Oral Implantology, vol. 4, pp. 11-29, 2011.

[9] T. von Arx and D. Buser, "Horizontal ridge augmentation using autogenous block grafts and the guided bone regeneration technique with collagen membranes: a clinical study with 42 patients," Clinical Oral Implants Research, vol. 17, no. 4, pp. 359-366, 2006.

[10] I. A. Urban, H. Nagursky, and J. L. Lozada, "Horizontal ridge augmentation with a resorbable membrane and particulated autogenous bone with or without anorganic bovine bonederived mineral: a prospective case series in 22 patients," The International Journal of Oral \& Maxillofacial Implants, vol. 26, no. 2, pp. 404-414, 2011.

[11] I. A. Urban, H. Nagursky, J. L. Lozada, and K. Nagy, "Horizontal ridge augmentation with a collagen membrane and a combination of particulated autogenous bone and anorganic bovine bone-derived mineral: a prospective case series in 25 patients," The International Journal of Oral \& Maxillofacial Implants, vol. 33, no. 3, pp. 299-307, 2013.

[12] C. H. F. Hämmerle, R. E. Jung, D. Yaman, and N. P. Lang, "Ridge augmentation by applying bioresorbable membranes and deproteinized bovine bone mineral: a report of twelve consecutive cases," Clinical Oral Implants Research, vol. 19, no. 1, pp. 19-25, 2008.

[13] L. Ohayon, "Ridge enlargement using deproteinized bovine bone and a bioresorbable collagen membrane: a tomodensitometric, histologic, and histomorphometric analysis," The International Journal of Periodontics \& Restorative Dentistry, vol. 31, no. 3, pp. 237-245, 2011. 
[14] S. S. Jensen and H. Terheyden, "Bone augmentation procedures in localized defects in the alveolar ridge: clinical results with different bone grafts and bone-substitute materials," The International Journal of Oral \& Maxillofacial Implants, vol. 24, pp. 218-236, 2009.

[15] A. Friedmann, K. Gissel, M. Soudan, B. M. Kleber, S. Pitaru, and T. Dietrich, "Randomized controlled trial on lateral augmentation using two collagen membranes: morphometric results on mineralized tissue compound," Journal of Clinical Periodontology, vol. 38, no. 7, pp. 677-685, 2011.

[16] P. A. Fugazzotto, "GBR using bovine bone matrix and resorbable and nonresorbable membranes. Part 1: histologic results," The International Journal of Periodontics \& Restorative Dentistry, vol. 23, no. 4, pp. 361-369, 2003.

[17] A. Patel, H. Greenwell, M. Hill, B. Shumway, and A. Radmall, "Ridge augmentation comparing an allograft plus autogenous bone chips to an osteoinductive demineralized bone Matrix," Implant Dentistry, vol. 28, no. 6, pp. 613-620, 2019.

[18] G. Mendoza-Azpur, A. de la Fuente, E. Chavez, E. Valdivia, and I. Khouly, "Horizontal ridge augmentation with guided bone regeneration using particulate xenogenic bone substitutes with or without autogenous block grafts: a randomized controlled trial," Clinical Implant Dentistry and Related Research, vol. 21, no. 4, pp. 521-530, 2019.

[19] M. Troeltzsch, M. Troeltzsch, P. Kauffmann et al., "Clinical efficacy of grafting materials in alveolar ridge augmentation: a systematic review," Journal of Cranio-Maxillo-Facial Surgery, vol. 44, no. 10, pp. 1618-1629, 2016.

[20] P. H. de Azambuja Carvalho, G. dos Santos Trento, L. B. Moura, G. Cunha, M. A. C. Gabrielli, and V. A. Pereira-Filho, "Horizontal ridge augmentation using xenogenous bone graft-systematic review," Oral and Maxillofacial Surgery, vol. 23, no. 3, pp. 271-279, 2019.

[21] L. Tolstunov, J. F. E. Hamrick, V. Broumand, D. Shilo, and A. Rachmiel, "Bone augmentation techniques for horizontal and vertical alveolar ridge deficiency in oral implantology," Oral and Maxillofacial Surgery Clinics of North America, vol. 31, no. 2, pp. 163-191, 2019. 